\title{
Check of the structure in photon pairs spectra at the invariant mass of about $38 \mathrm{MeV} / \mathrm{c}^{2}$
}

\author{
Khachik Abraamyan ${ }^{1,2, *}$, Chris Austin ${ }^{3}$, Mircea Baznat $^{4}$, Konstantin Gudima $^{4}$, \\ Mikhail Kozhin ${ }^{1}$, Sergey Reznikov ${ }^{1}$, and Alexander Sorin ${ }^{1,5}$ \\ ${ }^{1}$ VBLHEP JINR, 141980 Dubna, Moscow region, Russia \\ ${ }^{2}$ Yerevan State University, 0025, Yerevan, Armenia \\ ${ }^{3} 33$ Collins Terrace, Maryport, Cumbria CA15 8DL, England \\ ${ }^{4}$ Institute of Applied Physics, MD-2028 Kishinev, Moldova \\ ${ }^{5}$ BLTP JINR, 141980 Dubna, Moscow region, Russia
}

\begin{abstract}
Results of analysis of the effective mass spectra of photon pairs produced in $d \mathrm{C}, d \mathrm{Cu}$ and $p \mathrm{C}$ interactions at momenta of $2.75,3.83$ and $5.5 \mathrm{GeV} / c$ per nucleon, respectively, are presented. A structure at effective mass of about $38 \mathrm{MeV} / c^{2}$ is observed. The results of testing the observed signal are presented. The test results support the conclusion that the observed signal is the consequence of detection of a particle with a mass of about $38 \mathrm{MeV} / c^{2}$ decaying into a pair of photons.
\end{abstract}

\section{Introduction}

In recent years, a possibility of the existence of a light scalar - lighter than the known lightest hadron $-\pi^{0}$ meson, has been actively discussed. About the first indications of the existence of a $38 \mathrm{MeV}$ light scalar boson (henceforth referred to as $\mathrm{E}(38)$ ) and about their further discussions were reported in [1].

From the latest results, in particular, in [2], based on lattice simulations using highly improved staggered quarks for twelve-flavor QCD with several bare fermion masses, a flavorsinglet scalar state lighter than the pion in the correlators of fermionic interpolating operators is observed. The same state is also investigated using correlators of gluonic interpolating operators. Combined with their previous study, that showed twelve-flavor QCD to be consistent with being in the conformal window, the authors of [2] infer that the lightness of the scalar state is due to infrared conformality. This result shed some light on the possibility of a light composite Higgs boson ("technidilaton") in walking technicolor theories.

In [3], a hypothesis of existence of a scalar boson with the mass between about 100 $\mathrm{keV}$ and $100 \mathrm{MeV}$ which can simultaneously solve the proton radius puzzle and the muon anomalous magnetic moment discrepancy is proposed.

\footnotetext{
*e-mail: abraamyan@jinr.ru
} 


\section{Experiment}

\subsection{General layout}

The data acquisition of production of neutral mesons and $\gamma$-quanta in interactions of protons and light nuclei with nuclei has been carried out with internal beams of the JINR Nuclotron [4]. The experiments were carried out with internal proton beams at momentum $5.5 \mathrm{GeV} / c$ incident on a carbon target and with ${ }^{2} \mathrm{H},{ }^{4} \mathrm{He}$ beams and internal $\mathrm{C}-, \mathrm{Al}-, \mathrm{Cu}-, \mathrm{W}-, \mathrm{Au}-$ targets at momenta from 1.7 to $3.8 \mathrm{GeV} / c$ per nucleon. For the first analysis the data for the $d(2.0 A \mathrm{GeV})+\mathrm{C}, d(3.0 A \mathrm{GeV})+\mathrm{Cu}$ and $p(4.6 \mathrm{GeV})+\mathrm{C}$ reactions were selected. Some results on $\gamma \gamma$ pair production in these reactions, for the effective mass region, $M_{\gamma \gamma}>100 \mathrm{MeV} / c^{2}$ (photons in pair from different arms of the spectrometer) were reported in [4-6].

The presented data concern the reactions induced by protons and deuterons with momenta of 5.5, 2.75 and $3.83 \mathrm{GeV} / c$ per nucleon, respectively. Typical proton and deuteron fluxes were about $10^{8}$ and $10^{9}$ per pulse, respectively. The electromagnetic lead glass calorimeter PHOTON-2 was used to measure both the energies and emission angles of photons. The experimental instrumentation is schematically represented in Fig. 1. The PHOTON-2 setup is described in detail in [4]

The modules of the $\gamma$-spectrometer are assembled into two arms of 16 units. The modules in each arm are divided into two groups of eight units. The output signals of each group from eight counters are summed up linearly and sent to the inputs of four discriminators $\left(D_{i}\right)$. In these experiments all the discriminator thresholds were at the level of $0.4 \mathrm{GeV}$ in the $d(2.0 A \mathrm{GeV})+\mathrm{C}$ and $p(4.6 \mathrm{GeV})+\mathrm{C}$ experiments and at the level of $0.35 \mathrm{GeV}$ in the $d(3.0 A \mathrm{GeV})+\mathrm{Cu}$ experiment. Triggering takes place when there is a coincidence of signals from two or more groups from different arms: $\left(D_{1}+D_{2}\right) \times\left(D_{3}+D_{4}\right)$ in the $d(2.0 \mathrm{~A} \mathrm{GeV})+\mathrm{C}$ and $p(4.6 \mathrm{GeV})+\mathrm{C}$ experiments and $\left(D_{1}+D_{2}\right) \times\left(D_{3}+D_{4}\right) \times \overline{S 1} \times \overline{S 2}$ in the $d(3.0 A \mathrm{GeV})+\mathrm{Cu}$ experiment. In realizing the trigger conditions the amplitudes of all 32 modules were recorded on a disc. The dead time of data acquisition is about $150 \mu$ s per trigger. The mean rate of triggering was about $10^{3}$ events per spill. The duration of the irradiation cycle is $1 \mathrm{~s}$. Totally about $4.8 \times 10^{6}$ triggers were recorded during these experiments.

\subsection{Event selection}

To search for a signal at the low effective masses, we have analyzed photon pairs detected in the same arm of the $\gamma$-spectrometer. Below there are results of this analysis for photon pairs detected in the Right arm of the $\gamma$-spectrometer (situated at an angle of $26^{\circ}$, see Fig. 1). The expected width of the signal is defined by the uncertainties in the measured photon energies and in the opening angle of the photons. The errors of the measured opening angles were estimated from the analysis of simulated data, and are as follows: standard deviation, $\Delta \Theta \leq 0.9^{\circ}\left(\Delta \Theta \simeq 0.9^{\circ}\right.$ in cases where each photon triggers just one unit of the spectrometer, because the coordinates of a photon hit are determined more accurately, when the photon triggers several modules). The uncertainties $\Delta E_{\gamma}$ of the measured values of photon energies were estimated by the empirical formula for energy resolution averaged over the surface of the spectrometer [7, 9]:

$$
\Delta E_{\gamma} / E_{\gamma} \approx 0.068 / \sqrt{E_{\gamma}},
$$

where $E_{\gamma}$ is in $\mathrm{GeV}$. Distribution of the calculated value of $\Delta M_{\gamma \gamma}$ for the vicinity of the $\mathrm{E}(38)$ mass is shown in Fig. 2.

In order to identify the signal from detected particles, all photon pair combinations are used to calculate the invariant mass in each event. The combinatorial invariant mass spectrum of the $\gamma \gamma$ combinations (both photons from the Right arm of the spectrometer) is shown in 

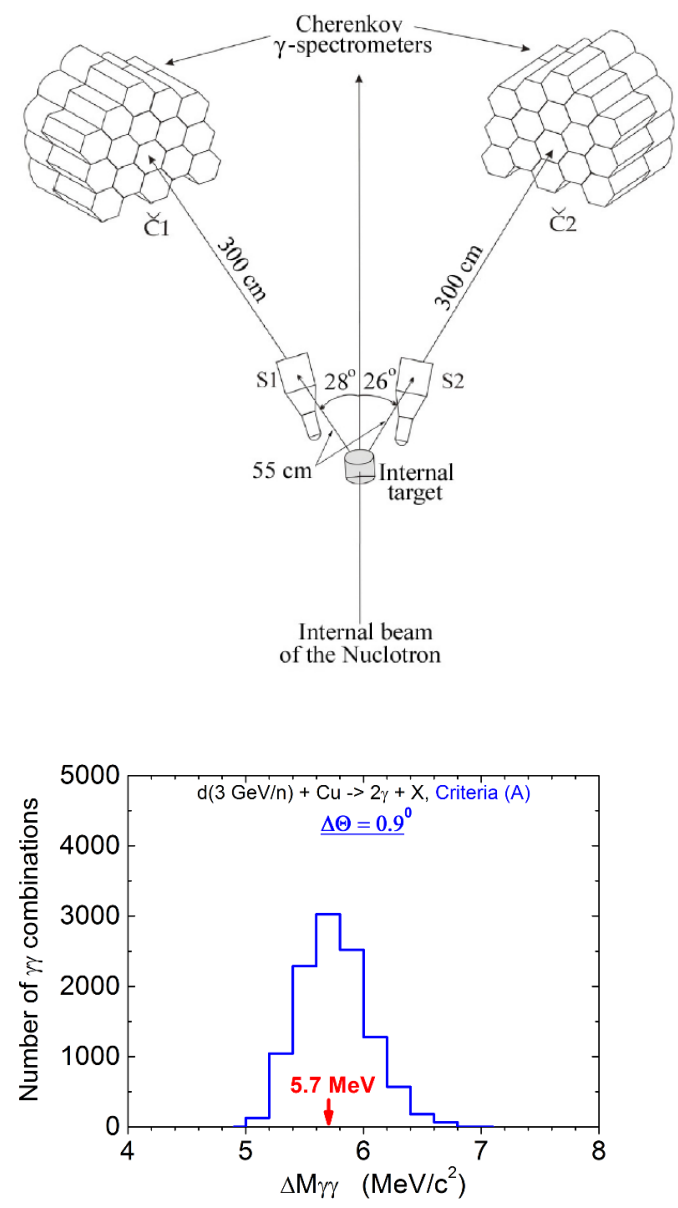

Figure 1. The schematic drawing of the experimental PHOTON-2 setup. The S1 and S2 are scintillation counters
Figure 2. Distributions of calculated value of $\Delta M_{\gamma \gamma}$ for photon pairs selected from the Right arm of the $\gamma$-spectrometer, under the Criteria (A) (see the text), for the vicinity of the $\mathrm{E}(38)$ mass: $36<M_{\gamma \gamma}<40 \mathrm{MeV} / c^{2}$, at the maximum value of $\Delta \Theta=0.9^{\circ}$

Fig. 3. The following selection criteria were used (criteria (A)): (i) the energies of photons, $E_{\gamma}>50 \mathrm{MeV}$ (practical boundary of workspace of the $\gamma$-spectrometer [7]); (ii) the opening angles of photons, $\operatorname{Cos} \Theta_{\gamma \gamma}<0.994$; (iii) the sum of the energies of two photons, $450<E_{12}<$ $750 \mathrm{MeV}$.

To see a possible structure of the invariant mass spectra, a background should be subtracted. The so-called event mixing method was used to estimate the combinatorial background: a photon in one event from the Right arm is combined with photon in other events from the same arm. In the mixing there are involved events with two or more photons in the Right arm satisfying criterion (i) and in the presence of one or more pairs of satisfying the condition (iii). This background was subtracted from the invariant mass distributions (see Fig. 3, bottom panels).

\section{Check of the observed peak}

Systematic errors may be due to uncertainty in measurements of $\gamma$ energies and inaccuracy in estimates of the combinatorial background. The method of energy reconstruction of events is described in detail in $[7,8]$. Possible overlapping effects were investigated previously for the reaction with the higher masses of the colliding nuclei and at higher energies in the reaction of $C+C$ at $4.5 \mathrm{GeV} / \mathrm{c}$ per nucleon [8]. It was found that the average displacement of the 

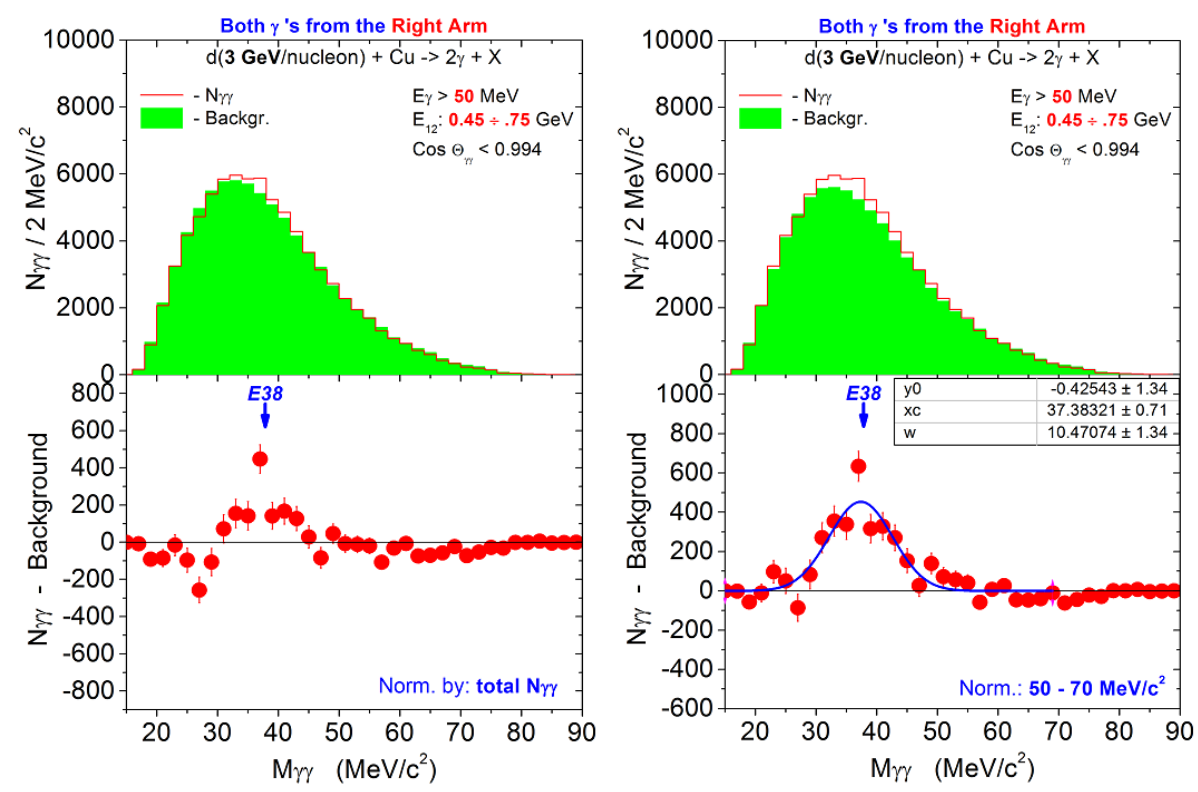

Figure 3. Invariant mass distributions (per $2 \mathrm{MeV} / \mathrm{c}^{2}$ ) of $\gamma \gamma$ pairs satisfying criteria (i)-(iii) (criteria (A)) without (upper panels) and with (bottom panels) the background subtraction obtained for the reaction $d+\mathrm{Cu} \rightarrow \gamma+\gamma+X$ at $3.83 \mathrm{GeV} / \mathrm{c}$ per nucleon. The top shaded histograms show the background contribution. The background is normalized to the total pair number (left) and to the number of pairs in a range of masses $50-70 \mathrm{MeV} / \mathrm{c}^{2}$ (right). The curve is the Gaussian approximation of the experimental points in the range $(14 ; 70) \mathrm{MeV} / \mathrm{c}^{2}$

effective masses of $\gamma \gamma$ pairs in the reaction is only $6 \%$. Thus, the influence of the overlap in the present experiment is negligible.

To elucidate the nature of the detected enhancement, at first we have investigated the dependence of its position on the energies of separate photons and photon pairs.

In addition to the above, we have also performed the following check of the stability of the observed structure:

- with or without the detected photons from different intervals of their energies and emission angles; in different intervals of opening angles of two photons;

- for pairs of photons inside different groups of the modules; in combinations of photons only from different groups;

- we have applied different cuts of pairs of photons, by the value of ratio of their energies $E_{\gamma_{1}} / E_{\gamma_{2}}$ (the latter can be successfully used in suppressing of the systematic errors).

Some example from this analysis is shown in Fig. 4.

Also, we have applied some methods of miscalibration of modules (by introducing the chaotic coefficients; with the extension of the standard error in the energies (amplitudes in each module) up to $2 \sigma, 4 \sigma$ etc), as well as different offsets of the energy scale.

All these results support the conclusion that the observed structure with an effective mass of about $38 \mathrm{MeV} / \mathrm{c}^{2}$ corresponds to the detection of particles with the given mass. 

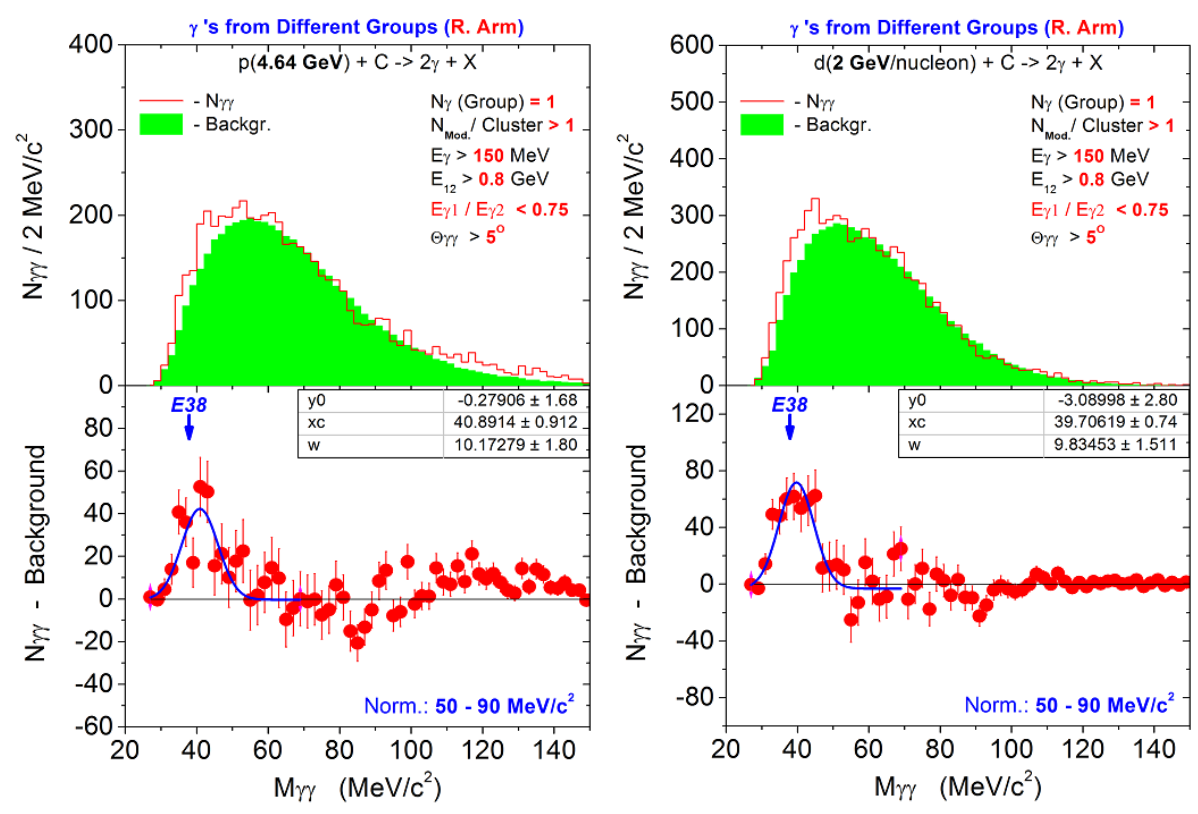

Figure 4. The same as in Fig. 3 but for $\gamma \gamma$ pairs selected from different groups (only 1 photon in each group, and when the photon triggers several modules), at $E_{\gamma \text { Min }}=150 \mathrm{MeV}$, at the sum of the energies of two photons, $E_{12}>0.8 \mathrm{GeV}$, at the ratio of their energies $E_{\gamma_{1}} / E_{\gamma_{2}}<0.75$

\section{Data simulation}

In order to verify the experimental method of allocation of the $\mathrm{E}(38)$ signal there were simulations of the studied reaction performed with accurate experimental measurement conditions, including the set of the corresponding event class, criteria and efficiency of photon pair energy and effective mass measurement, background determination and subtraction.

To simulate the $d+\mathrm{Cu}$ reaction, we have used a transport code described in detail in [4].

As the first step, any sources of $\mathrm{E}(38)$ were not included in the model. Calculations showed good reproduction of the spectra of photons produced in the reaction and the spectrum of effective masses of two photons under the real conditions of the experiment. At the same time, there are no resonant structures in the spectrum of the effective mass of two photons in the region with expected appearance of the signal from the $\mathrm{E}(38)$. This result can serve as a confirmation of the chosen experimental techniques and basic components incorporated in the model reaction. At the same time it can serve as proof that none of the recorded sources of photons or a combination of photons from different sources may lead to a resonant structure in the expected region of effective masses.

Then we included the second step, for which we used a simplified model in which the $\mathrm{E}(38)$ boson interacts equally with all quark types through a single Yukawa coupling constant $g$, and does not interact directly with any other Standard Model particles. (The E(38) decays to two photons would thus occur mainly through a one-loop process involving the light quarks) 


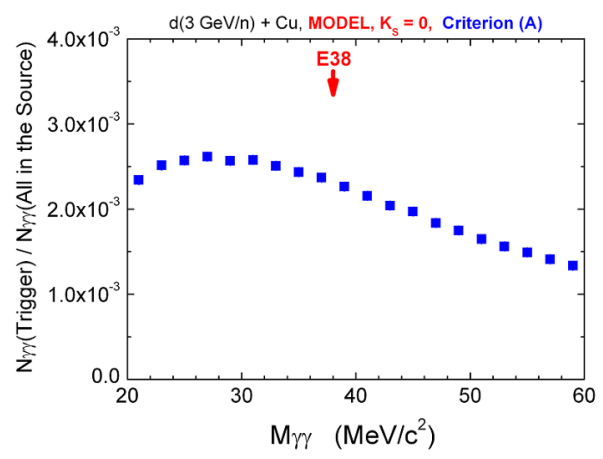

Figure 5. The ratio of the number of triggered $\gamma \gamma$ pairs under real experimental conditions (at $E_{\gamma}>50 \mathrm{MeV}$ (criterion (A))) on the total number in the simulated source, depending on the effective mass (per $2 \mathrm{MeV} / \mathrm{c}^{2}$ ) of $\gamma \gamma$ pairs for the Model after excluding the events with $\gamma \gamma$ pairs from the $\mathrm{E}(38)$ boson
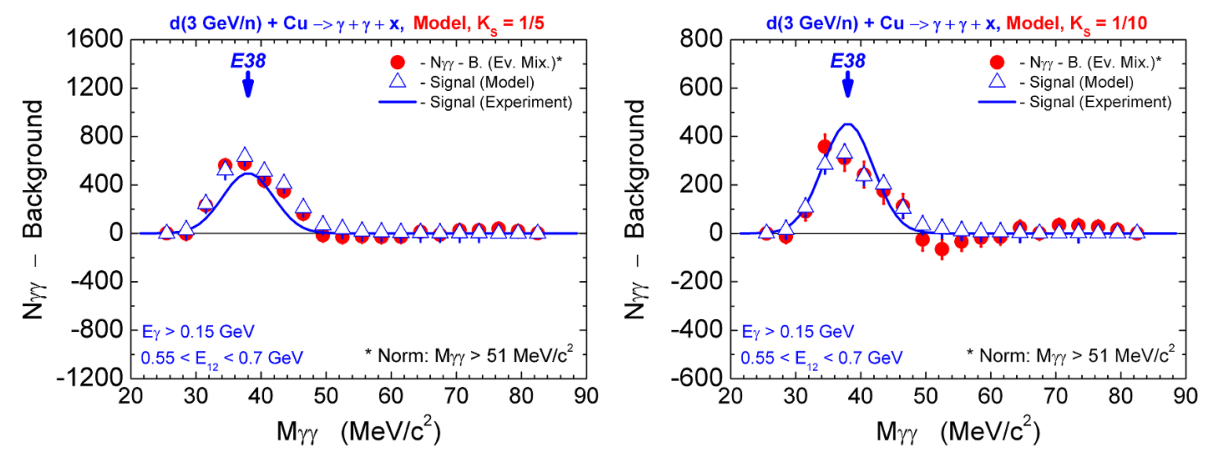

Figure 6. Invariant mass distributions (per $3 \mathrm{MeV} / c^{2}$ ) of $\gamma \gamma$ pairs from the decay of $\mathrm{E}(38)$ under the real experimental conditions: $\Delta$ - pairs $\gamma \gamma$, selected by means of labels of photons applied in the model; $\bullet$ the result of processing the simulated data with the method applied to the experimental data. The curves are the experimental signal after the background subtraction (Gaussian approximation) proportional to the number of $d+\mathrm{Cu}$ events containing a pair of $\gamma \gamma$ in the required energy range. The signal in the simulated data reduced by a factor $K_{S}=1 / 5$ (among the events, containing a photon from the decay of $\mathrm{E}(38)$, each 5 th event was taken) (left figure) and by a factor $K_{S}=1 / 10$ (right figure)

\subsection{Detection efficiency of photon pairs}

The relative efficiencies of $\gamma \gamma$-pairs detection and selection for the Right arm of the $\gamma$ spectrometer, at different effective masses of $\gamma \gamma$-pairs, are shown in Fig. 5. In this analysis, the coincidence with the Left arm is not required (i.e. triggering takes place when there is a signal from one or two groups from the Right arm).

As is seen from Fig. 5 the detection efficiency is quite smoothly distributed with respect to $M_{\gamma \gamma}$.

\subsection{Checking the signal extraction accuracy}

To check the accuracy of the signal extraction, we have applied different factors to reduce the signal in the simulated data. Some spectra after the signal reduction are given in Fig. 6.

Acknowledgements. We are grateful to S. Gevorgyan, M. Kapishin, V. Kekelidze, A. Litvinenko, D. Madigozhin, V. Nikitin and Yu. Potrebenikov for numerous fruitful discussions and hope for further joint work. We thank S. Afanasev, A. Elishev, A. Kovalenko, A. Malakhov, and the staff of the Nuclotron for their help in carrying out the experiments. 


\section{References}

[1] E. van Beveren, G. Rupp, arXiv:1102.1863v2. E. van Beveren, S. Coito, G. Rupp, EPJ Web Conf. 95, 02007 (2015). [arXiv:1411.4151]

[2] Yasumichi Aoki et al., arXiv:1305.6006v2

[3] Yu-Sheng Liu, David McKeen, and Gerald A. Miller, Phys. Rev. Lett. 117, 101801 (2016)

[4] Kh. U. Abraamyan et al., Phys. Rev. C 80, 034001 (2009). Doklady Physics 55, 161 (2010). Phys. of Part. and Nucl. 41, 1097 (2010). Phys. Atom. Nucl. 75, 657 (2012)

[5] Kh. U. Abraamyan et al., Eur. Phys. J. A 52, 259 (2016)

[6] Kh. Abraamyan et al., EPJ Web Conf. 138, 04006 (2017)

[7] Kh. U. Abraamyan et al., Instruments and Experimental Techniques 32, 58 (1989). 39, 775 (1996)

[8] Kh. U. Abraamyan et al., Phys. Lett. B 323, 1 (1994). Phys. Atom. Nucl. 59, 252 (1996). 60, 1843 (1997). 68, 982 (2005). EPJ Web Conf. 138, 05003 (2017)

[9] M.N. Khachaturian, JINR Communication E1-85-55, Dubna (1985) 